Tropical Journal of Pharmaceutical Research April 2010; 9 (2): 197-203

(C) Pharmacotherapy Group,

Faculty of Pharmacy, University of Benin

Benin City, 300001 Nigeria.

All rights reserved.

Research Article

Available online at http://www.tjpr.org

\title{
Development of Chitosan Acetate Films for Transdermal Delivery of Propranolol Hydrochloride
}

\section{KSY Hemant* and HG Shivakumar}

Department of Pharmaceutics, J.S.S. College of Pharmacy, JSS University, S.S. Nagar, Mysore-570 015, Karnataka, India.

\begin{abstract}
Purpose: To formulate and evaluate chitosan acetate films designed for transdermal delivery of propranolol hydrochloride.

Methods: Chitosan acetate was chemically modified with acetaldehyde and the solution was prepared with $1 \%$ acetic acid, in which was dissolved propranolol hydrochloride, was cast as films in Petri dish and characterised by Fourier transform infrared spectroscopy (FTIR), differential scanning calorimetry (DSC) and nuclear magnetic resonance (NMR). The films were evaluated for permeability, swelling, and in vitro drug release.

Results: Drug content of propranolol hydrochloride in the films ranged from 0.9 to $1.4 \mathrm{mg} / \mathrm{cm}^{2}$. Swelling was $570 \%$ for chitosan acetate and $180 \%$ for chitosan while drug release through chitosan acetate higher than through chitosan. Permeability coefficient was $6.12 \times 10^{-4}$ and $0.97 \times 10^{-4} \mathrm{~g}_{\mathrm{cm}} / \mathrm{c}$ day for chitosan acetate and chitosan, respectively. FTIR and DSC results indicated that there was no chemical interaction between the drug and the polymers used. NMR spectra showed the appearance of specific peaks for acetate group. Differences between chitosan acetate and chitosan were significant $(p<0.05)$ with regard to permeability, swelling and in vitro drug release.

Conclusion: The films prepared using the synthesised chitosan acetate exhibited superior physicochemical and drug release characteristics to those of chitosan. The results also indicate chitosan acetate films may be suitable for delivering propranolol hydrochloride via the transdermal route which offers some advantages over other routes.
\end{abstract}

Keywords: Chitosan, Chitosan acetate, Films, Transdermal route, Propranolol hydrochloride, Permeability. 


\section{INTRODUCTION}

Chitosan is a linear, semi-rigid polysaccharide, which is believed to be a promising biomaterial in view of its biodegradability, biocompatibility and relatively low toxicity. Chitosan is a copolymer of N-acetyl D-glucosamine and Dglucosamine. Chemical modification has been attempted to improve water solubility and bioactives of the polymer[2]. To improve its aqueous solubility many derivatives of chitosan have been synthesised,e.g., carboxymethyl chitosan which exhibits better solubility and moisture retaining property than chitosan [1]. Many chitosan derivatives have been found to have antibacterial activity and can be used as such, e.g., hydroxypropyl chitosan [2].

Chitosan is a weak base and a certain amount of acid is required to transform the glucosamine units into the uncoiled and positively charged water-soluble form [3]. At neutral $\mathrm{pH}$ values, the chitosan molecule will loose its charge and therefore precipitate from solution [3]. In this regard possibilities for the potential use of chitosan as an absorption enhancer in the more basic environment of the large intestine, colon and rectal mucosa are limited. However, potential reactive groups make chitosan a versatile polymer with unique properties. Chitosan derivatives with different physico-chemical properties, especially water solubility at neutral and basic conditions, will be of particular interest, e.g., N-trimethyl chitosan chloride is able to increase the transport of large hydrophilic compounds [3]. The charge density and structural features of this derivative of chitosan makes it to act as a absorption enhancer across mucosal epithelia by opening the tight junctions [3]. Chitosan acatate exhibits better micromeritic properties than the pharmaceutical binder, PVP K30, and as a tablet binder, it could produce sustained drug release [4].

Chemical modifications of chitosan have been reported. These include interaction with salicylic acid when incorporated into chitosan films using acetic acid as a solvent/vehicle; this, in turn, affected its drug release behavior [5]. Spray dried chitosan microspheres, loaded with insulin for protein delivery, prepared using acetic acid as solvent, also showed chenial interaction with the protein [6]. Furthermore, studies have been reported whereby chitosan was modified to $\mathrm{N}$-trimethyl chitosan chloride, carboxymethyl chitosan and hydroxyl propyl chitosan [2,3].

Chronic diseases, such as asthma and rheumatoid arthritis, if treated via the transdermal route, may offer several advantages over other routes such as prevention of first pass metabolism and high bioavailability. However delivery of drugs through transdermal drug delivery system is limited due to limited absorption through the skin. Films produced by casting chitosan solution, when applied to skin, often cause irritation due to the presence of traces of acid except the films are washed first to remove traces of acid. Propranolol hydrochloride, which was selected for this study, has a biological half life of $4 \mathrm{~h}$ and is, therefore, usually administered several times a day. Consequently, there is a need to develop a dosage form which delivers the drug in a sustained fashion. Propranolol hydrochloride is subject to an extensive and highly variable hepatic first pass metabolism following oral administration with a reported systemic bioavailability of $15-23 \%$, indicating the need for alternative drug delivery modes. Hence, in this study, an attempt has been made to prepare chitosan acetate transdermal films for the delivery of propranolol hydrochloride..

\section{EXPERIMENTAL}

\section{Materials}

Propranolol hydrochloride was a gift from M/s Strides Arcolab Ltd, Bangalore. Chitosan was procured from Vishu Aquatech, Madras, India. All other reagents used were of analytical grade. 


\section{Preparation of chitosan and chitosan acetate solution}

Chitosan solution $(2 \% \mathrm{w} / \mathrm{v})$ was prepared by stirring chitosan in $1 \%$ acetic acid using a homogeniser until the chitosan dissolved completely. Chitosan acetate was prepared by mixing $50 \mathrm{ml}$ of the chitosan solution with $2 \mathrm{ml}$ of acetaldehyde by stirring continuously for $3 \mathrm{~h}$ at $60{ }^{\circ} \mathrm{C}$. The solution was cooled and acetone was added to precipitate chitosan acetate [7].

\section{Fabrication of films}

Propranolol hydrochloride [400 mg] was added to $100 \mathrm{ml}$ of either chitosan or chitosan acetate solution prepared above and stirred with a homogeniser for half an hour to dissolve the drug. The mixture was poured into Petri dish and dried overnight at $40{ }^{\circ} \mathrm{C}$. The films produced were peeled from from the Pteri dish and washed with $50 \%$ ethanol to remove surface-bound traces of acid.

\section{Assessment of drug entrapment}

Films of $1 \mathrm{~cm}^{2}$ in area were weighed and placed in a $100 \mathrm{ml}$ volumetric flask containing $50 \mathrm{ml}$ of distilled water and kept for $24 \mathrm{~h}$. The volume was made up to $100 \mathrm{ml}$ with distilled water, filtered and the drug content was assayed by measuring the absorbance at 290 nm using UV spectrophotometer (Schimadzu 1700 ) and that of a standard over a concentration range of $10-40 \mu \mathrm{g} / \mathrm{ml}$. The test was carried out in triplicate.

\section{Water absorption (Swelling) Studies}

A film of $1 \mathrm{~cm}^{2}$ in area was weighed and placed in a watch glass containing approximately $10 \mathrm{ml}$ of water. At regular intervals, the membrane was taken out, blotted with filter paper and weighed on a digital balance. After attaining equilibrium weight, the membranes were dried and weighed to determine \% water absorbed which was taken as \% swelling. All tests were in triplicate.

\section{Permeability studies}

Approx. $1 \mathrm{~g}$ of calcium chloride was taken in dried vials $(2.3 \times 4.8 \mathrm{~cm})$ and the films $(1$ $\mathrm{cm}^{2}$ ) were tightly fixed over the brim of the vials with the aid of an adhesive. The vials were accurately weighed and kept in a desiccator containing a saturated solution of potassium chloride at room temperature. The vials were removed and weighed daily for 7 days. Permeability or water vapor transmission rate (WVTR) for chitosan and chitosan acetate films were calculated formulas in Eq 1.

$W V T R=W L / S$

where $W$ is the weight of water transmitted, $L$ the thickness of the film and $S$ the film surface area [7]. The tests were performed in triplicate.

\section{In vitro drug release studies}

In vitro drug release was assessed in distilled water by means of a Franz diffusion cell with the magnetic stirrer set at $100 \mathrm{rpm}$. The amount of drug released was determined by withdrawing $5 \mathrm{ml}$ of the dissolution fluid at various time intervals over $24 \mathrm{~h}$. The volume withdrawn was replaced with an equal volume of fresh distilled water to maintain sink conditions. The withdrawn samples were analysed spectrophotometrically at $290 \mathrm{~nm}$ using distilled water as blank [10]. All determinations were in triplicate.

\section{Fourier transform infra-red spectroscopy (FTIR)}

FTIR analysis of both chitosan and chitosan acetate was performed on a Shimadzu FTIR $8400 \mathrm{~S}$ using $\mathrm{KBr}$ pellet to hold the sample and was expressed in $\mathrm{cm}^{-1}$. The solid pellet was prepared using $\mathrm{KBr}$ at room temperature. 


\section{Differential scanning calorimetry (DSC)}

DSC thermograms and thermal transition temperatures for chitosan and chitosan acetate were obtained and examined using a differential scanning colorimeter (DSC Q 100, TA Instruments) to determine whether there was any interaction between the drug and the polymers used. Sample size was 2 - $4 \mathrm{mg}$ and the samples were sealed in aluminium pans and heated at a rate of $5{ }^{\circ} \mathrm{C} / \mathrm{min}$ in a nitrogen gas atmosphere.

\section{Nuclear magnetic resonance (NMR)}

${ }^{13} \mathrm{C}$ NMR studies were carried out on the films with an NMR spectrometer (DSX-300, Bruker, India) in the solid state at $75 \mathrm{Mhz}$ to determine whether there were chemical interactions between the drug and the polymers.

\section{Data analysis}

To determine the mechanism of drug release from the films, the release data were fitted to zero-order, first-order, and Higuchi models using the software PCP DissoV-2.08 software (Poona College of Pharmacy, Pune, India) Comparison of the data obtained for chitosan and chitosan acetate was made by Student $\mathrm{t}-$ test at $95 \%$ level of confidence using Microsoft Excel 2007.

\section{RESULTS}

\section{Drug entrapment}

The content of propranolol hydrochloride in the films was 0.9 and $1.4 \mathrm{mg} / \mathrm{cm}^{2}$ for chitosan and chitosan acetate, respectively.

\section{Water absorption (swelling)}

As Fig 1 shows, the films prepared with chitosan acetate showed nearly 3 times greater swelling than chitosan. Swelling was as high as 570 and $180 \%$ for chitosan acetate and chitosan, respectively.
Table 1: Swelling ( $\%, \pm S D)$ of chitosan and chitosan acetate films $(n=3)$

\begin{tabular}{ccc}
\hline Time (h) & $\begin{array}{c}\text { Chitosan } \\
\text { film }\end{array}$ & $\begin{array}{c}\text { Chitosan } \\
\text { acetate } \\
\text { film }\end{array}$ \\
\hline 1 & $20.25 \pm 0.43$ & $53.21 \pm 0.56$ \\
2 & $26.43 \pm 0.63$ & $76.21 \pm 1.19$ \\
3 & $34.35 \pm 1.32$ & $132.12 \pm 2.34$ \\
4 & $55.56 \pm 1.34$ & $189.43 \pm 0.57$ \\
5 & $75.12 \pm 0.32$ & $275.54 \pm 1.19$ \\
6 & $89.98 \pm 2.32$ & $332.18 \pm 1.21$ \\
7 & $118.67 \pm 2.54$ & $402.84 \pm 0.57$ \\
8 & $139.75 \pm 1.21$ & $473.21 \pm 1.12$ \\
9 & $164.67 \pm 0.57$ & $506.67 \pm 2.32$ \\
10 & $180.46 \pm 2.50$ & $570.31 \pm 1.19$ \\
\hline
\end{tabular}

\section{Water vapor transmission rate}

The water vapor transmission (or permeability) data showed that films prepared from chitosan acetate exhibited moisture transmission of $6.12 \times 10^{-4} \mathrm{~g} . \mathrm{cm}^{2} /$ day, compared to $0.97 \times 10^{-4} \mathrm{~g} . \mathrm{cm}^{2} /$ day for chitosan. Thus, chitosan acetate films were approx. six times more permeable than chitosan films.

\section{In vitro drug release}

Fig 1 shows the in vitro drug release pattern of chitosan and chitosan acetate films. The data indicate that drug release from chitosan acetate films was higher $(p<0.05)$ than from chitosan films. The drug release data of both chitosan and chitosan acetate films showed a good fit to the Higuchi model with $r^{2}$ value of 0.9952 and 0.9981 , respectively.

\section{FTIR spectroscopy}

The FTIR spectra of chitosan and chitosan acetate, shown in Fig 2, exhibited broad peaks in the range of $3450-3400 \mathrm{~cm}^{-1}$. These peaks were assigned to $\mathrm{OH}$ stretching, indicating intermolecular hydrogen bonding. The spectra also overlapped in the same region of $\mathrm{NH}$ stretching. The $\mathrm{C}-\mathrm{O}$ stretching 


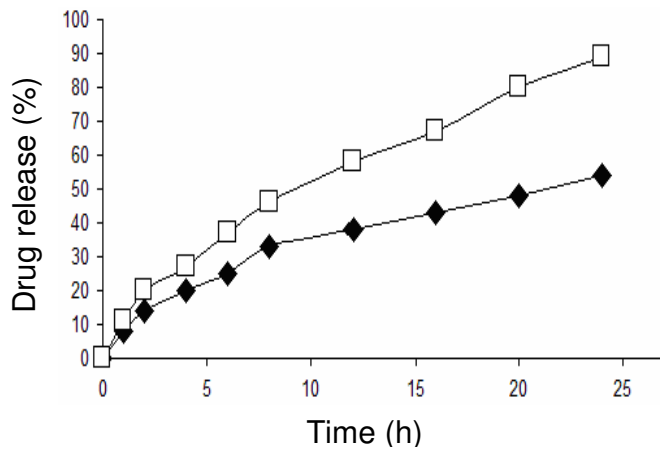

Figure 1: In vitro release pattern of chitosan $(\square)$ and chitosan acetate ( $\square$ ) films

peak near $1655 \mathrm{~cm}^{-1}$ (representing the structure of $\mathrm{N}$-acetylglucosamine) as well as the $\mathrm{NH}_{2}$ stretching peak at $1600 \mathrm{~cm}^{-1}$ (representing the glucosamine functional group) were exhibited in the spectrum of chitosan. In the spectrum of chitosan acetate, the peak near $1655 \mathrm{~cm}^{-1}$ disappeared but a strong peak at $1550-1600 \mathrm{~cm}^{-1}$ and a weak peak near $1400 \mathrm{~cm}^{-1}$ region appeared which are attributed to asymmetric and symmetric carboxylate anion stretching. These peaks provide evidence of the conversion of chitosan to chitosan acetate.

\section{DSC}

The DSC thermograms in Fig 3 indicate conversion of chitosan to the acetate derivative did not affect its thermal properties as the decomposition temperature of chitosan and chitosan acetate remained stable at 87.7 ${ }^{0} \mathrm{C}$.

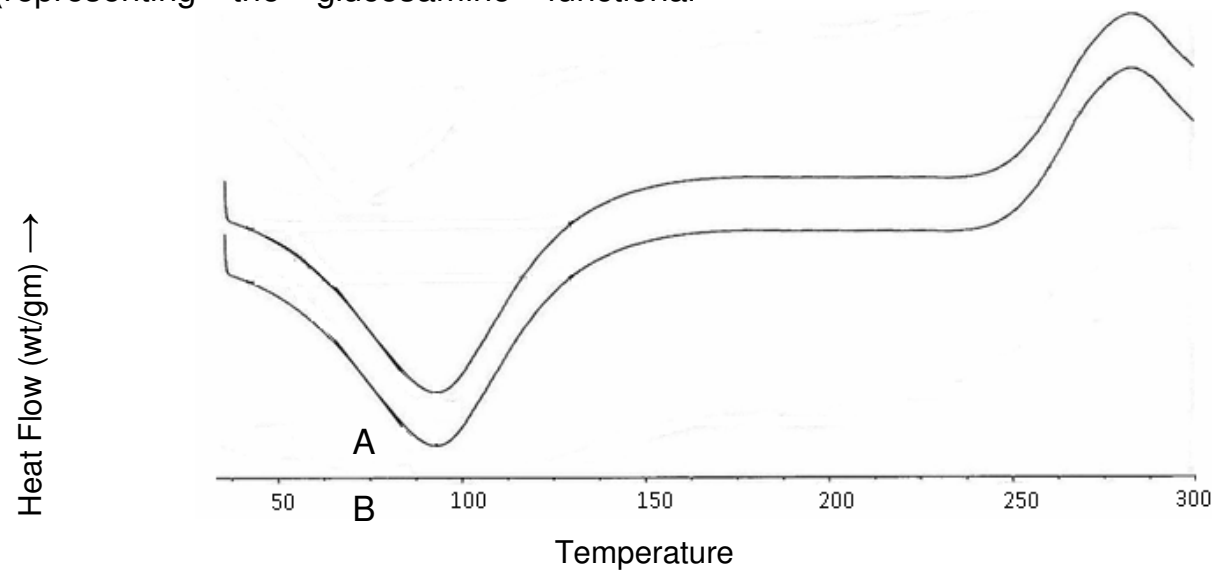

Figure 3: DSC spectra of chitosan (A) and chitosan acetate (B)

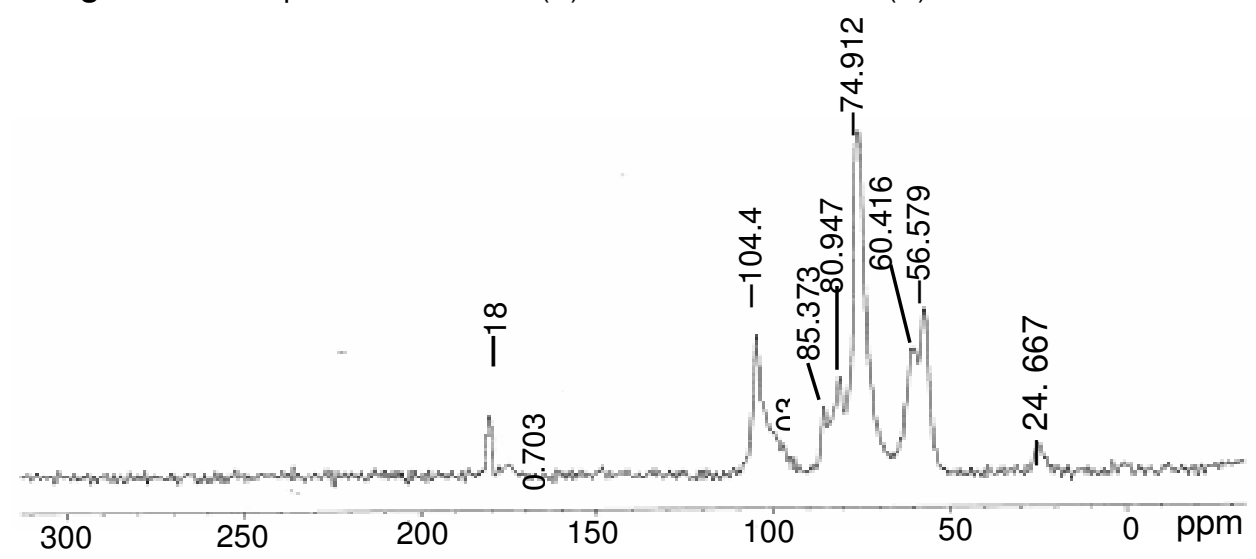

Figure 4: NMR spectra of chitosan acetate 


\section{NMR spectroscopy}

Examination of the ${ }^{13} \mathrm{C}$ NMR spectrum of chitosan acetate shows resonance at $50-$ $110 \mathrm{ppm}$ which was attributed to the presence of glocosamine units. The resonance at $180 \mathrm{ppm}$ was assigned to carbonyl carbon while the one at 24ppm was assigned to $\mathrm{CH}_{3}$ carbon which represents acetate functionality. As a result of the high degree of deacetylation of chitosan, the resonance assigned to $\mathrm{CH}_{3}$ carbon at 24 ppm, $\mathrm{N}$ acetyl glucosamine units at $174 \mathrm{ppm}$, and carbonyl carbon at $180 \mathrm{ppm}$ could hardly be observed in chitosan spectra [4] while these were clearly visible in chitosan acetate spectra.

\section{DISCUSSION}

To form chitosan acetate, the free amino group of chitosan reacted with an aldehyde in the presence of acid to form Schiff's base. Acetaldehyde was selected based on its filmforming capability with chitosan. Chemicallymodified chitosan is capable of producing membranes with superior properties such as high efficiency in drug loading, permeability and drug release due to changes in the polymeric interpenetrating network in the grafted polymer.

Various films have been used in the delivery of various drugs as transdermal delivery systems $[7,15]$. From the results obtained, it is clear that propranolol hydrochloride was loaded into the transdermal film in desired amount to give therapeutic levels of the drug. The swelling and permeability (WVTR) of films were good as documented in the results section while release studies showed that the drug was released in a sustained manner over $24 \mathrm{~h}$ with up to $80 \%$ of the loaded drug released.

Drug loading was higher for chitosan acetate due to the formation of Schiff's bases with acetaldehyde which generated more space within the polymeric network for trapping the drug. Since chitosan is insoluble in water, the films did not dissolve but swelled, but swelling was greater for chitosan acetate films due to its loose polymeric network. The formation of Schiff's base with acetaldehyde and the consequent generation of larger space within the polymeric network explain why chitosan acetate films showed higher permeability than chitosan films. This also explains why drug release was higher for chitosan acetate than chitosan films.

FTIR spectra support the claim that chitosan was actually converted to chitosan acetate and that the main peaks remained the same without any change in their respective wavelengths. The DSC thermograms also buttress the fact that there was no chemical interaction between the drug and the polymers used while ${ }^{13} \mathrm{C}$ NMR spectra confirmed that chitosan was converted into chitosan acetate. The high linearity of the regression coefficient, when the release data were fitted to the Higuchi model, indicate that drug release was matrix diffusion-controlled. This resulted in sustained release of the drug from the films over a period of $24 \mathrm{~h}$.

\section{CONCLUSION}

Chitosan acetate has some advantages over chitosan including higher drug entrapment, greater water permeability and swelling, and faster in vitro drug release. These advantages should make chitosan acetate films suitable for propranolol hydrochloride delivery via the transdermal route, a route which offers several advantages over other routes.

\section{ACKNOWLEDGEMENT}

The authors thank the J.S.S. Mahavidyapeetha, Mysore and JSS University, Mysore, India, for their valuable support in the course of this research. The authors also wish to thank Strides Arcolab Ltd, Bangalore and IISC, Bangalore, India for making available their facilities for DSC and NMR studies, respectively. 


\section{REFERENCES}

1. Lingyun $C$, Yumin $D$, Huiqing $W$, Ling $X$. Relationship between molecular structure and moisture retention ability of carboxymethyl chitin and chitosan. J Appl Polym Sci 2002; 83: 1233-1241.

2. Wenming X, Peixin X, Wei W, Qing L. Preparation and antibacterial activity of a water soluble chitosan derivative. Carbohydrate Polymers 2002; 50: 35-40.

3. Kotze A, Lueben H, Bas L, Hans J. Comparison of the effect of different chitosan salts and $\mathrm{N}$ trimethyl chitosan chloride on the permeability of intestinal epithelial cells. J Control Rel. 1998; 51: 35-46.

4. Nunthanid J, Laungtanaanan $M$, Sriamornsak $P$. Characterization of chitosan acetate as a binder for sustained release tablets. J. Control. Rel. 2004; 99: 15-26.

5. Puttipipatkhachorn S, Nunthanid J, Yamamoto G. Drug physical state and drug polymer interaction on drug release from chitosan matrix films. J. Control. Rel. 2001; 75: 143-153.

6. Genta L, Pavanetto F, Conti B. Improvment of Dexamethasone dissolution rate from dried chitosan microspheres. S.T.P Pharm. Sci. 1995; 5: 202-207.

7. Kulkarni V, Keshavayya J, Shastri C. Transdermal Delivery of Antiasthmatic Drug through Modified Chitosan Membrane. Indian J. Pharm. Sci. 2005; 67: 544-547.

8. Majeti $N$, Kumar $R$. A review of chitin and chitosan applications. React. Func. Polymers 2000; 46 : 1-27.

9. Hejazi $R$, Amiji M. Chitosan based gastrointestinal delivery systems. J. Control. Rel. 2003; 89: 151165.

10. Silvia R, Giuseppina S, Franca F. Buccal delivery of acyclovir from films based on chitosan and polyacrylic acid. Pharm. Dev Tech. 2003; 8: 199208.

11. Youwen L, Qing C, Hongbing L. Preparation and characterization of $\mathrm{N}$-(2-carboxybenzyl)chitosan as a potential $\mathrm{pH}$-sensitive hydrogel for drug delivery. Carbohyd Res 2007; 342: 87-95
12 .Jian $D$, Jing $D$, Jun-Long $L$, Theresa $D$. Novel $p H$ sensitive polyelectrolyte carboxymethyl Konjac glucomannan-chitosan beads as drug carriers. React Func Polymers 2006; 66: 1055-1061.

13. Colo G, Zambito Y, Burgalassi S. Effect of chitosan and of $\mathrm{N}$-carboxymethylchitosan on intraocular penetration of topically applied ofloxacin. Int $J$ Pharm 2004; 273: 37-44.

14. Eddy C, Antonio I, Bernard B. Development and optimization of a novel sustained-release dextran tablet formulation for propranolol hydrochloride. Int J Pharm 2006; 317: 32-39.

15. Nagarajan M, Narmada S, Ganesham M. Preparation and evaluation of transdermal patches of salbutamol sulphate. Eastern Pharmacist 1996; 29: 127-128.

16. Jurairat $N$, Kampanart $H$, Manee L. Development of time, $\mathrm{pH}$ and enzyme-controlled colonic drug delivery using spray-dried chitosan acetate and hydroxypropyl methylcellulose. Eur J. Pharm. Biopharm. 2008; 68: 253-259.

17. Yan L, Xi G, Nan L. Physicochemical characterization and antibacterial property of chitosan acetates. Carbohyd Polymers 2007; 67: 227-232.

18. Ping $D$, Ke-Long $H$, Gui-Yin $L$. Preparation and properties of modified chitosan as potential matrix materials for drug sustained-release beads. Int. J. Bio. Macromol. 2007; 131: 125-131.

19. Ajit $R$, Namdev S, Sangamesh P, Tejraj M. Novel interpenetrating polymer network microspheres of chitosan and methylcellulose for controlled release of theophylline. Carbohyd Polymers 2007; 69: 678-687.

20. Athena W, Merrill D, David M. Metal complexation of chitosan and its glutaraldehyde cross-linked derivative. Carbohyd Res 2007; 342: 1189-1201.

21. Maher Z, Entsar S, Khaled S, Mohamed E. Surface modification of polypropylene films by chitosan and chitosan/pectin multilayer. Carbohyd Polymers 2008; 71:187-195.

22. Mara E, Maria V, Helio C. Supercritical solvent impregnation of ophthalmic drugs on chitosan derivatives. J Supercritical Fluids 2008; 44: 245 257.

23. Genta L, Pavanetto F, Conti B. Improvment of Dexamethasone dissolution rate from dried chitosan microspheres. S.T.P Pharm. Sci. 1995; 5: 202-207. 